\author{
P. G. MEZGER \\ Max-Planck-Institut für Radioastronomie, Bonn, D.B.R.
}

\begin{abstract}
This paper deals with the interpretation of molecular line emission from class $\mathrm{I} \mathrm{OH} / \mathrm{H}_{2} \mathrm{O}$ emission centers associated with compact $\mathrm{HII}$ regions and with the $\mathrm{OH} 18 \mathrm{~cm}$ emission from dark clouds in T-Tauri star associations. Observational evidence is presented, that class $\mathrm{I} \mathrm{OH} / \mathrm{H}_{2} \mathrm{O}$ emission centers represent a particular stage in the evolution of a massive star (or a group of massive stars) whereas protostars of lower mass apparently do not go through such a stage.

It appears that in associations the low-mass stars are formed first and the massive $O$-stars are formed last. T-Tauri star associations may represent an early evolutionary stage of a star association where low-mass stars are formed. Evidence is presented that the physical conditions in some parts of the Taurus complex of dust clouds and T-Tauri stars are appropriate for the formation of single stars of about a solar mass.
\end{abstract}

\title{
1. Review of Earlier Work
}

Quite inadvertently the $\mathrm{OH} 18 \mathrm{~cm}$ line was found in strong emission close to galactic $\mathrm{H}$ II regions. At about the same time the first systematic surveys were made of radio recombination lines which are emitted by HII regions. The close correlation in the radial velocities of $\mathrm{OH}$ and recombination lines showed, that the positional coincidence was not fortuitious but that $\mathrm{HII}$ regions and $\mathrm{OH}$ emission centers were, in fact, closely associated in space (Mezger and Höglund, 1967).

It soon became clear that the $\mathrm{OH}$ emission centers had extremely small angular dimensions and that, therefore, incredibly high brightness temperatures were required to account for the observed line flux density. These characteristics made $\mathrm{OH}$ emission centers ideal objects for interferometry, and soon the CalTech and MIT groups (Cudaback et al., 1966; Rogers et al., 1966; Raimond and Eliasson, 1969) provided $\mathrm{OH}$ positions which were by orders of magnitude better than those of the associated HII regions. To match this positional accuracy Schraml and Mezger (1969) made a survey of most of the northern HII regions with high surface brightness, using the NRAO $140 \mathrm{ft}$ telescope at the wavelength $1.95 \mathrm{~cm}$. An angular resolution of $2^{\prime}$ and a positional accuracy of about $30^{\prime \prime}$ was achieved. At about the same time Ryle and Downes (1967) applied, for the first time, the aperture synthesis technique to the observation of a galactic Hil region, DR 21, in the Cygnus X region. Both groups independently discovered the existence of a new class of compact HıI regions with high electron densities and small linear dimensions (Mezger et al., 1967). The combination of our radio observations with optical observations led us to the conclusion, that these compact $\mathrm{HII}$ regions were the very early evolutionary stages of stellar subgroups first discovered by Blaauw (1964) in nearby OB-associations. This in turn led us to the hypothesis that the process of formation of massive stars was responsible for the $\mathrm{OH}$ emission (Mezger et al., 1967). It was previously thought that pumping of the $\mathrm{OH}$ maser by UV radiation supplied by the HII regions and their exciting stars was the link between $\mathrm{H}$ II regions and $\mathrm{OH}$ sources. Models of non- 
thermal $\mathrm{OH}$ and $\mathrm{OH} / \mathrm{H}_{2} \mathrm{O}$ emission respectively by protostars were described in two previous papers (Mezger and Robinson, 1968; Litvak, 1969).

\section{Star Formation}

This paper deals with class $\mathrm{I} \mathrm{OH} / \mathrm{H}_{2} \mathrm{O}$ emission centers associated with compact $\mathrm{HII}$ regions and with the quasi-thermal emission of the central $\mathrm{OH} 18 \mathrm{~cm}$ lines from dust clouds, especially from dust clouds which are associated with T-Tauri stars. The common link of both phenomena is the process of star formation. Therefore a brief outline of our present ideas how stars form out of the interstellar matter will be presented.

For the formation of stars, a certain volume of the interstellar space must become gravitationally unstable and contract. Gravitational contraction may be initiated by a density wave, which compresses the interstellar matter. Presumably this is the mechanism for the formation of Population I stars in genuine spiral arms. Gravitational contraction may also be initiated by a decrease of temperature and internal turbulence of the interstellar matter, for example as the result of increased cooling by an increased production of molecules and dust. This latter mechanism appears to pertain to the formation of stars in regions outside regular spiral arms.

Most of our present knowledge of star formation in clusters and associations is based on optical observations, which do not pertain to regular spiral arms. I therefore do not know, if the following picture also applies to the process of star formation by a density wave in regular spiral arms.

Optical observations and their theoretical interpretation (Iben and Talbot, 1966; Williams and Cremin, 1969) have shown, that in nearby associations stars of about one solar mass are formed first and the most massive O-stars are formed last. O-stars, and their associated stellar subgroups, appear to form out of clouds of some thousand solar masses (Blaauw, 1964). Once the O-stars reach the main sequence (MS) they ionize the remnant of the proto-cluster and star formation in this subgroup comes to a halt. This remnant appears to be tightly packed around the O-stars, in the form of a shell or cocoon, and the ionized gas is therefore first observed as a very compact HII region of high density, which subsequently rapidly expands.

The Trapezium cluster is the youngest of four stellar subgroups in the Orion association. It is associated with the compact $\mathrm{HII}$ region M 42. About $3-5 \times 10^{5} \mathrm{yr}$ ago the Trapezium cluster apparently started to expand (Strand, 1958). At about the same time the Trapezium O-stars must have been formed out of a dense cloud located at the center of the Trapezium cluster. They reached the MS about $1.6 \times 10^{4} \mathrm{yr}$ ago, as estimated from their dynamical age (Strand, 1970, private communication). At the same time the compact HII region M 42 must have formed, and its age of 1.4 to $2.3 \times 10^{4} \mathrm{yr}$, derived by Vandervoort (1964) agrees in fact perfectly with the dynamical age of the Trapezium stars. This picture leads to two predictions which are of importance in the context of this paper: there must be regions of star formation where predominantly low-mass stars are formed, embedded in which are dense clouds, out of which eventually an O-star or a close group of O-stars will form. 


\section{Class I OH/ $/ \mathrm{H}_{2} \mathrm{O}$ Sources}

After this digression into the general problem of star formation let me come back to the proper topic of my paper, i.e. molecular lines emitted from dense clouds in regions of star formation.

Class I OH sources are those whose center lines at 1665 and $1667 \mathrm{MHz}$ are greatly enhanced by some maser mechanism. They are always associated with HII regions, and the close correlation in radial velocities shows that this is not a projection effect. The apparent diameters of the individual $\mathrm{OH}$ emission centers are very small, corresponding to typical linear dimensions of some $10^{14} \mathrm{~cm}$. The maser amplification may be as high as $10^{13}$. Emission of the $\mathrm{H}_{2} \mathrm{O} 1.35 \mathrm{~cm}$ line has been found close to the $\mathrm{OH}$ class I emission centers. The pioneering work of the MIT VLBI group has shown, that the apparent size of the $\mathrm{H}_{2} \mathrm{O}$ emission centers is by an order of magnitude smaller than that of the associated $\mathrm{OH}$ emission centers (Burke et al., 1970). Other characteristics of the $\mathrm{H}_{2} \mathrm{O}$ emission are very similar to that of class I OH emission centers. Nearly all the strong $\mathrm{OH}$ emission centers are associated with $\mathrm{H}_{2} \mathrm{O}$ emission which, however, is considerably stronger than the corresponding $\mathrm{OH}$ emission. Cases where no $\mathrm{H}_{2} \mathrm{O}$ emission has been detected from class $\mathrm{I} O \mathrm{OH}$ sources may well be a result of the high system noise of the present $\mathrm{H}_{2} \mathrm{O}$ radiometers. According to Turner and Rubin (1970, private communication) there is at present only one $\mathrm{H} I \mathrm{l}$ region, G $34.3+0.1$, known, where no $\mathrm{OH}$ emission has been found from an $\mathrm{H}_{2} \mathrm{O}$ emission center.

One of the most obvious characteristics of class $\mathrm{I} \mathrm{OH} / \mathrm{H}_{2} \mathrm{O}$ emission centers is their association with compact $\mathrm{H}$ II regions, which appear to be the ionized cocoons of recently formed O-stars. I made a careful reinvestigation of the nature of this association of $\mathrm{OH} / \mathrm{H}_{2} \mathrm{O}$ emission centers and compact $\mathrm{HII}$ regions, based on recent $2 \mathrm{~cm}$ single dish observations (Churchwell et al., 1969) and on aperture synthesis observations made by the NRAO and Cambridge groups, respectively. There are clear-cut cases like the two $\mathrm{OH} / \mathrm{H}_{2} \mathrm{O}$ emission centers associated with NGC 6334 or the two emission centers north of DR 21 in the Cygnus $X$ region, where the emission centers may be embedded in an extended low-density $\mathrm{H}$ II region, but where the angular separation from the associated compact HII regions is $3^{\prime}$ or more. This is a confirmation of our earlier conclusion (Mezger et al., 1967), that there is no physical connection between $\mathrm{OH} / \mathrm{H}_{2} \mathrm{O}$ emission centers and compact $\mathrm{H}$ II regions. How then shall we explain, that these emission centers are always found in the vicinity of compact $\mathrm{HII}$ regions? I suggest that compact $\mathrm{HII}$ regions and $\mathrm{OH} / \mathrm{H}_{2} \mathrm{O}$ emission centers represent different stages in the evolution of O-stars. Formation of O-stars, on the other hand, requires very special conditions of the interstellar matter, which prevail in an association only for a very limited time. And this, I feel, is the reason why compact $\mathrm{HII}$ regions and class $\mathrm{I} \mathrm{OH} / \mathrm{H}_{2} \mathrm{O}$ emission centers are found to be associated.

It is important to realize, that obviously only the massive stars go through the stage of strong $\mathrm{OH} / \mathrm{H}_{2} \mathrm{O}$ masering. In further support of this statement note, that 


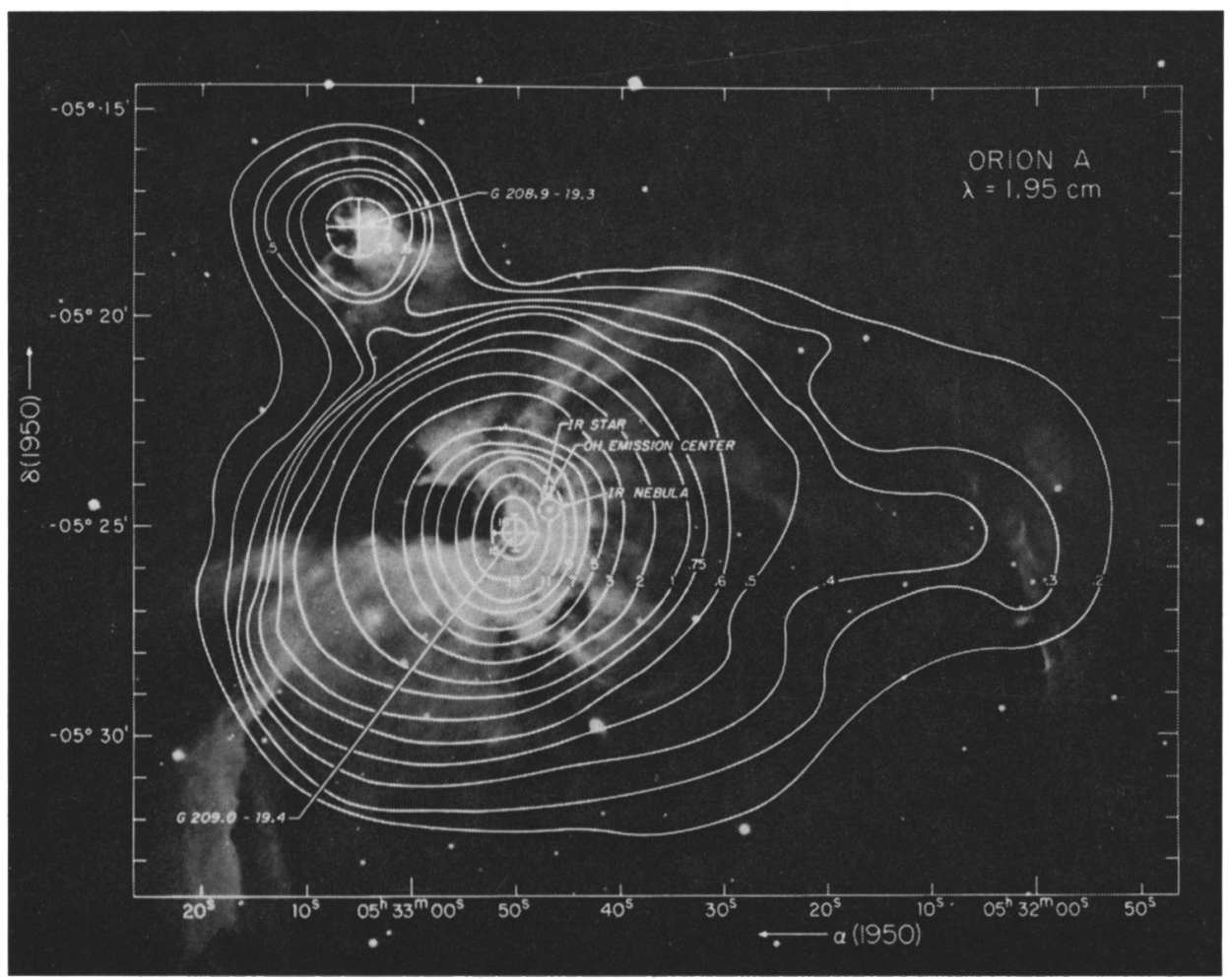

Fig. 1. Overlay of radio contours of the Orion Nebula on an $\mathrm{H} \alpha$ photograph. Observed at $15.4 \mathrm{GHz}$ with an angular resolution of $2^{\prime}$ (Schraml and Mezger, 1969).

although formation of low-mass stars in T-Tauri associations goes on for a long time, no strong $\mathrm{OH} / \mathrm{H}_{2} \mathrm{O}$ emission has been detected in these regions (Ball, 1970, private communication; Turner, 1969). There are other cases like M 42, IC 1795/W 3 and W 49, where single dish continuum observations do not exclude the possibility of a coincidence of $\mathrm{OH} / \mathrm{H}_{2} \mathrm{O}$ emission center and compact $\mathrm{H}$ II region. Only aperture synthesis observations can bring a decision. I will leave $\mathrm{W} 49$ out of this discussion; with a distance of about $14 \mathrm{kpc}$ this giant HII region is too far away. The case of M 42, the compact $\mathrm{H}$ II region associated with the Trapezium cluster (Figure 1), I have discussed in two papers last year (Mezger, 1970a, b). Although the $\mathrm{OH} / \mathrm{H}_{2} \mathrm{O}$ emission center lies within the boundaries of the compact Hil region, the aperture synthesis map by Webster and Altenhoff (1970) shows no conspicuous feature in the free-free emission at the general position of the emission centers. This can be explained in two ways: either the molecules are formed and emit in the Hil region proper; or the positional coincidence is a mere projection effect. For reasons which I will state later I believe in this latter explanation. I suggest that this association of $\mathrm{OH} / \mathrm{H}_{2} \mathrm{O}$ emission centers with an IR nebula and an IR star north-west of the Trapezium is the fifth and youngest subgroup of the Orion association, where the 
O-stars are in the process of formation but have not yet reached the MS. In another $10^{4} \mathrm{yr}$, the present compact $\mathrm{HII}_{\mathrm{I}}$ region $\mathrm{M} 42$ will have evolved into a low-density HII region, but another compact Hil region may be seen at the location of the $\mathrm{OH} / \mathrm{H}_{2} \mathrm{O}$ emission centers.

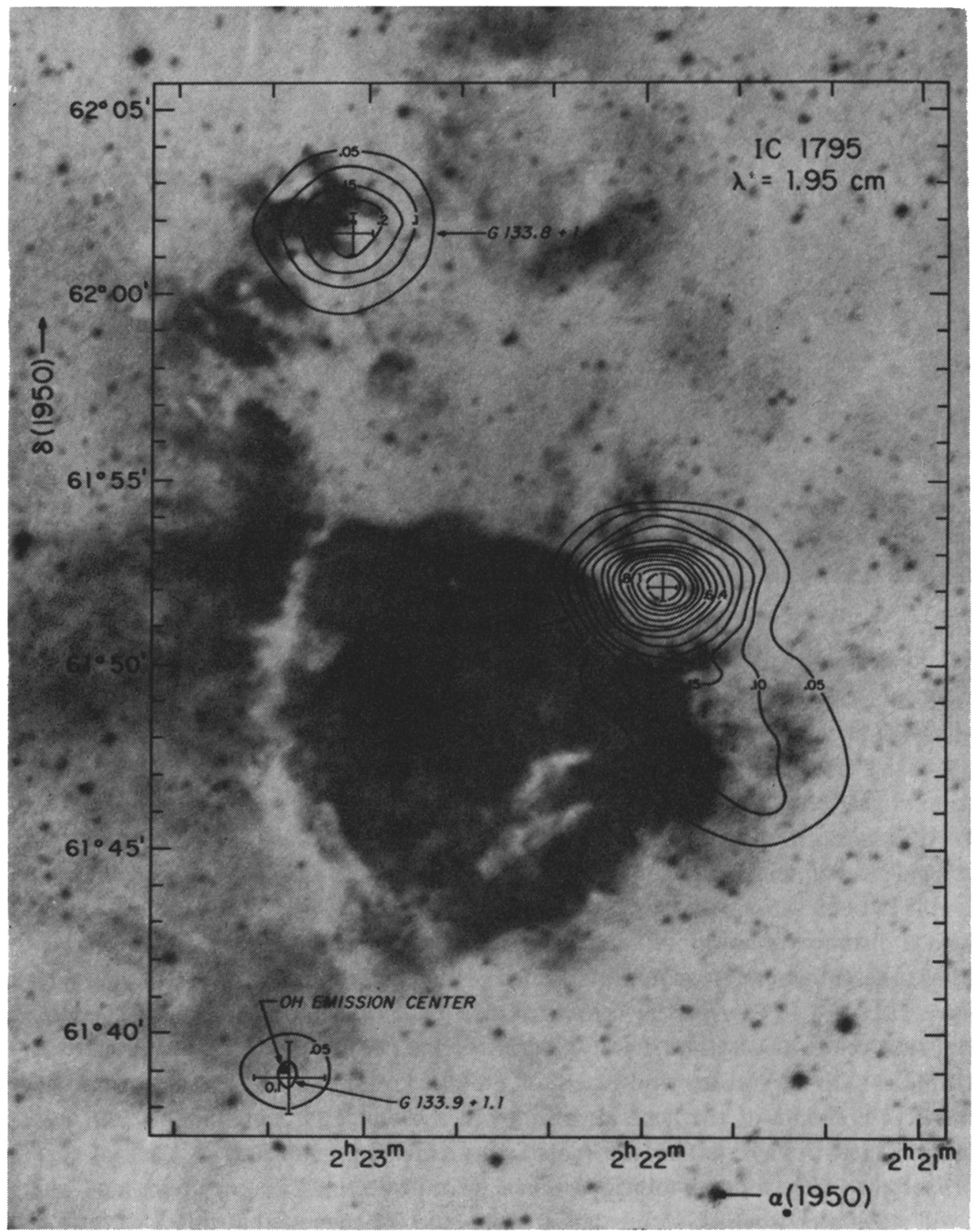

Fig. 2. Overlay of radio contours of the thermal source W 3 on an $\mathrm{H} \alpha$ photograph of IC 1795 . Observed at $15.4 \mathrm{GHz}$ with an angular resolution of $2^{\prime}$ (Schraml and Mezger, 1969). 
IC 1795/W3 appears to me the most relevant case and I will therefore discuss it at more length. IC 1795 is probably the youngest of three optically visible HII regions in the Perseus arm, the others being IC 1805 and 1848, respectively. Figure 2 shows an overlay of $2 \mathrm{~cm}$ radio contours on an $\mathrm{H} \alpha$ photograph of IC 1795. The main radio component G $133.7+1.2$ of W 3 lies to the west of IC 1795. In my opinion this compact giant $\mathrm{Hu}$ region is not part of the optically visible HiI region IC 1795 but rather represents the fourth and youngest in this sequence of spiral arm HiI regions.

$\mathrm{OH} / \mathrm{H}_{2} \mathrm{O}$ emission has first been detected from a point about $17^{\prime}$ southeast of the main component of W 3 . Subsequently, by observations in the short centimetre wavelength continuum, one of the most compact and therefore, probably youngest $\mathrm{HII}$ regions, G $133.9+1.1$, was found to coincide with the $\mathrm{OH}$ emission center (Mezger et al., 1967; Aikman, 1968). More recently, both $\mathrm{H}_{2} \mathrm{O}$ and weak $\mathrm{OH}$ emission was also found close to the main radio component $\mathrm{G} 133.7+1.2$.

The southern compact component, G $133.9+1.1$ was observed by Wynn-Williams, using the Cambridge aperture synthesis telescope at $5 \mathrm{GHz}$, where the source is still optically thick. He derived its position $\left(\alpha=2^{\mathrm{h}} 23^{\mathrm{m}} 16.48^{\mathrm{s}} ; \delta=61^{\circ} 38^{\prime} 56.8^{\prime \prime}\right)$ with an accuracy of $0.5^{\prime \prime}$. Adopting an electron temperature of $10^{4} \mathrm{~K}$ and a flux density of $3 \mathrm{fu}$ at $15 \mathrm{GHz}$, he estimates an apparent diameter of $1.7^{\prime \prime}$, corresponding to a linear diameter of $6.5 \times 10^{16} \mathrm{~cm}$. Density and mass of this compact HII region are then found to be $N_{\mathrm{e}}=2 \times 10^{5} \mathrm{~cm}^{-3}$ and $M_{\mathrm{HII}} / M_{\odot}=0.04$, respectively (Wynn-Williams, private communication). The excitation parameter is about $38 \mathrm{pc} \mathrm{cm}^{-2}$, corresponding to an exciting star of spectral type $\mathrm{O}$, if the compact $\mathrm{H}$ II region is ionization bounded.

Interferometer positions of the $\mathrm{OH}$ emission center have been obtained by Raimond and Eliasson (1969). The more accurate $1665 \mathrm{MHz}$ position is $\alpha=2^{\mathrm{n}} 23^{\mathrm{m}} 16.8^{\mathrm{s}} \pm 0.2^{\mathrm{s}}$ and $\delta=61^{\circ} 38^{\prime} 54^{\prime \prime} \pm 1^{\prime \prime}$. Moran et al. (1968) have resolved the $1665 \mathrm{MHz}$ emission source into at least seven individual emission centers of a typical linear size of about $2 \times 10^{14} \mathrm{~cm}$, which are distributed in an area of size $1.2^{\prime \prime} \times 2.3^{\prime \prime}$. The centers of $\mathrm{OH}$ and free-free emission are hence separated by about $3^{\prime \prime}$, which is about 2.5 times the mean positional uncertainty. A positional coincidence of compact $\mathrm{H}$ II region and $\mathrm{OH}$ emission center cannot be ruled out completely; however, it appears highly unlikely.

Again, I suggest, that we are observing here two O-stars (or close groups of $\mathrm{O}$ stars) in different evolutionary stages. The northern O-star has just reached the MS and is starting to ionize the remnants of the protostar. The southern O-star is in an earlier evolutionary stage. The solid angle from which $\mathrm{OH}$ emission is observed has about the same size as the solid angle subtended by the adjacent compact Hu region. This makes me believe that the seven emission centers resolved by VLBI observations pertain to one single massive protostar. With an apparent diameter of $2.3^{\prime \prime}$ and a total mass of, say, $50 \mathrm{M}_{\odot}$, the average density of this protostar would be $2 \times 10^{6}$ atoms $\mathrm{cm}^{-3}$, and its diameter would be $9 \times 10^{16} \mathrm{~cm}$, corresponding to the early stages of free-fall contraction of a protostar (Hayashi and Nakano, 1965).

Maser action would be seen whenever the physical conditions along the line of sight are appropriate. It was objected that the differential velocity of a protostar in free-fall contraction would limit the pathlength of the maser severely. Dr Nakano 
(1970, private communication) has kindly computed the velocity field of a contracting protostar of $10 \mathrm{M}_{\odot}$ and of radius $3.7 \times 10^{16} \mathrm{~cm}$. For polytropic indices $n=0$ and 1.5 the free-fall velocity changes from $0 \mathrm{~km} \mathrm{sec}^{-1}$ at the center to less than $3 \mathrm{~km} \mathrm{sec}^{-1}$ in the outer layers of the contracting protostar. For polytropic index $n=4$, the freefall velocity attains a maximum of about $5.25 \mathrm{~km} \mathrm{sec}^{-1}$ at 0.17 times the radius of the protostar and subsequently decreases monotonically to $2.7 \mathrm{~km} \mathrm{sec}^{-1}$ in the outermost layers. Differential velocity along the line of sight therefore does not appear to be a severe limitation to maser action. On the other hand, especially if combined with a rotation of the protostar, one can conveniently explain the velocity range of 7.4 $\mathrm{km} \mathrm{sec}^{-1}$ covered by the seven individual emission centers.

Does $\mathrm{OH}$ and $\mathrm{H}_{2} \mathrm{O}$ masering occur in the same volume of space? Interferometer positions for the $\mathrm{H}_{2} \mathrm{O}$ emission centers are still lacking. But there is other observational evidence that $\mathrm{OH}$ and $\mathrm{H}_{2} \mathrm{O}$ masering does not occur in exactly the same regions of the protostar. The $\mathrm{OH}$ and $\mathrm{H}_{2} \mathrm{O}$ emission spectra usually cover the same velocity range, but there is no one-to-one correspondence in the individual emission spikes, whose widths - if interpreted as thermal Doppler broadening - correspond to kinetic temperatures of about $20 \mathrm{~K}$ for the $\mathrm{OH}$ and several $100 \mathrm{~K}$ for the $\mathrm{H}_{2} \mathrm{O}$. If these lines were produced by an unsaturated maser they would be narrowed and the kinetic temperature could go up by a factor of, say, twenty. But even then at least the $\mathrm{OH}$ temperatures would be considerably lower than that of a typical Hil region. This is another argument that $\mathrm{OH} / \mathrm{H}_{2} \mathrm{O}$ emission comes from dense neutral condensations.

We can estimate upper and lower limits for the density range of these condensations. The fact that Doppler broadening appears to dominate over collisional broadening yields upper limits of $3 \times 10^{14}$ and $2 \times 10^{15}$ atom $\mathrm{cm}^{-3}$ for the density of $\mathrm{OH}$ and $\mathrm{H}_{2} \mathrm{O}$ emission centers, respectively. A lower limit of the density can be derived from the condition that the molecules must be shielded against photo-ionization and dissociation. Effective shielding of the Lyman continuum radiation in the vicinity of an $\mathrm{O}$-star requires densities of $N_{\mathrm{H}} \gtrsim 10^{4} \mathrm{~cm}^{-3}$. Effective shielding against $U V$ radiation longward of the Lyman continuum limit requires densities of $N_{\mathrm{H}} \gtrsim 10^{6} \mathrm{~cm}^{-3}$.

I have deliberately not touched upon the subject of the total mass involved in the $\mathrm{OH} / \mathrm{H}_{2} \mathrm{O}$ masers. Such an estimate involves the esoteric process of pumping of the molecules which will be dealt with in a subsequent panel discussion. And it involves an estimate of both the number of oxygen atoms tied up in $\mathrm{OH}$ and $\mathrm{H}_{2} \mathrm{O}$ molecules and the geometry of the maser. But a straight forward estimate, based on the number of emitting molecules, leads usually to masses of the masering volumes ranging from sub-stellar to stellar masses. I don't think that other estimates, based on improved pumping models, will come up with radically different answers.

In summary I conclude, that the physical conditions derived for class $\mathrm{IOH} / \mathrm{H}_{2} \mathrm{O}$ emission centers are compatible with the hypothesis that this emission comes from proto-stars. The association of $\mathrm{OH} / \mathrm{H}_{2} \mathrm{O}$ emission centers with compact $\mathrm{H}$ II regions on the one hand, absence of strong class $\mathrm{I} \mathrm{OH} / \mathrm{H}_{2} \mathrm{O}$ emission from T-Tauri star associations on the other hand indicates, that only the very massive protostars evolve through the stage of class $\mathrm{I} \mathrm{OH} / \mathrm{H}_{2} \mathrm{O}$ emission centers. 


\section{Dark Clouds and T-Tauri Associations}

One of the most striking features of all molecular lines is their correlation in both position and radial velocity with galactic Hil regions. Galactic Hir regions, on the other hand, are found in those regions of our Galaxy where the neutral hydrogen (HI) attains its maximum surface density and presumably also its highest space density (Kerr et al., 1968; Mezger et al., 1969). In other words, stars appear to be formed in regions of high density of the neutral interstellar gas. In the context of this review paper we are interested in two problems: (1) What do O-star associations look like at the time when the low-mass stars only are present and the O-stars still wait for their formation. (2) Is the formation of dust and molecules a consequence of the formation of low-mass stars as suggested by Herbig (1970); or is star formation rather initiated by an increased production of dust and molecules and a subsequent increased cooling of the gas in dense clouds. The obvious place where low mass stars are formed in large quantities and over a large volume of space are T-Tauri associations. T-Tauri stars are only found in or near regions of significant dust concentration and it is now recognized that the T-Tauri stars are formed out of the dust clouds in which they are found. The large volume covered by some of the T-Tauri associations speaks against a process of gravitational collapse of a large cloud and subsequent dispersal of the stars so formed. It is clear in some observed cases (Herbig, 1970) that single T-Tauri stars have been formed from small discrete dust clouds; therefore, we have to face the question of how single stars of about $1 M_{\odot}$ can form. The answer is that, for a minimum temperature of the interstellar gas of $3 \mathrm{~K}$, and a gas density of $10^{4}$ atom $\mathrm{cm}^{-3}$, the Jeans's mass is about $1.6 \mathrm{M}_{\odot}$.

I first use molecular line emission to probe, if conditions in dark clouds and T-Tauri associations are compatible with the formation of single stars. In some dark clouds $\mathrm{OH}$ emission has been observed and these lines can be used to estimate the temperatures. Cudaback and Heiles (1969) derive temperatures between 6 and $9 \mathrm{~K}$ for four clouds where the $\mathrm{OH}$ lines are seen in absorption against background continuum radiation. Assuming LTE emission for the two central OH lines, Heiles (1969) derived temperatures from 4.4 to $10 \mathrm{~K}$. Turner (1970, private communication) quotes even lower temperatures of $3.6 \mathrm{~K}$ for the Taurus cloud, and $5.4 \mathrm{~K}$ for the Ophiuchus cloud. Densities of dust clouds can only be inferred by adopting a value for the mass absorption coefficient. Heiles (1970, private communication) estimates densities between $10^{2}$ and $10^{3}$ atom $\mathrm{cm}^{-3}$, which are probably underestimates since not all heavy elements are tied up in grains and the grains are probably not of optimum size. In fact, if the temperature of the interstellar gas is to be lower than about $11 \mathrm{~K}$, it must be shielded against both subcosmic particles and the UV radiation longward of the Lyman continuum which can ionize carbon and metals (Hjellming, 1970, private communication). Werner (1970) finds, that such shielding is effectively achieved for clouds of densities $>10^{4}$ atom $\mathrm{cm}^{-3}$. Thus, temperatures and densities within the dark clouds appear to be very close to those required for collapse of $1 M_{\odot}$ stars. However, in the above estimate $I$ applied the Jeans's criterion assuming no turbulence. 
Line widths observed for $\mathrm{OH}$ are typically of the order of $1-2 \mathrm{~km} \mathrm{sec}^{-1}$. These usually resolve into two or more components, with widths of about $0.75 \mathrm{~km} \mathrm{sec}^{-1}$. This corresponds to thermal velocities in a cloud with a temperature of $200 \mathrm{~K}$, and it is clear that most of the observed line widths must be due to internal mass motions. These motions may explain why low-mass stars form more easily than high mass stars, a fact not explained by the Jeans's criterion alone. It is possible that $1 M_{\odot}$ stars form in small regions in the cloud where the relative motions are low, while the probability of finding a volume in such a cloud containing $10 M_{\odot}$ and more in which relative motions are negligible may be very small.

The largest account of data relating to dark clouds and the associated stars is that for the Taurus complex, so I will refer mainly to that region; sparser evidence for other regions indicates that the same conditions apply. Figure 3 shows the area of the Taurus complex. Absorption of greater than 1 magnitude is extensive; the contours derived by McCuskey (1938) are shown. Within the general absorption are many small regions of extremely heavy absorption - probably greater than 5 magnitudes (Heiles (1968) estimates 8 magnitudes for the center of the largest cloud in Taurus). Those heavily obscured areas that have been observed for OH (Heiles, 1970; Heiles, 1968; Cudaback and Heiles, 1969) are indicated by hatching; most of these regions are too small to affect McCuskey's large scale contours (in many cases the clouds

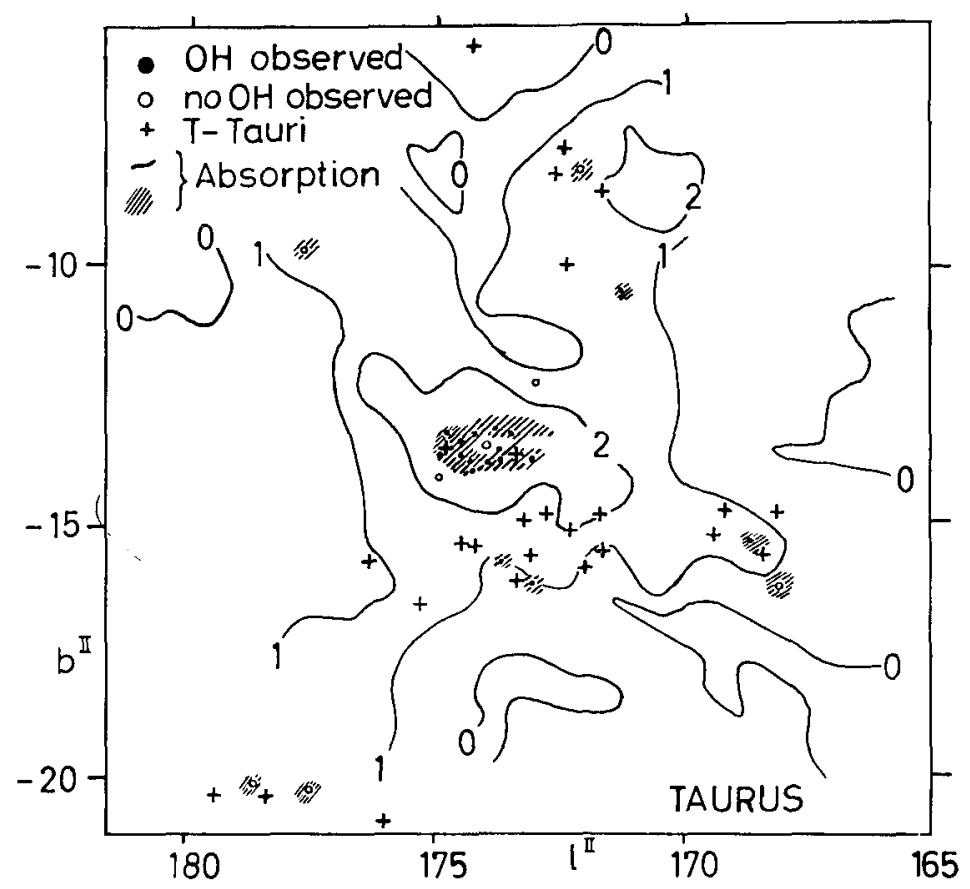

Fig. 3. The relative distribution of $\mathrm{OH}$ sources, T-Tauri stars and visual absorption in the region of Taurus. 
measure only about $10 \mathrm{~min}$ arc, and are therefore even smaller than the hatched areas on the diagram). Positive results for the $\mathrm{OH}$ observations are indicated by filled circles, negative results are indicated by open circles. Positions of T-Tauri stars, as given by Herbig (1962) are indicated by crosses. They are found in large numbers throughout the Taurus complex - within regions where the absorption is 1.5 magnitude or greater.

Quasi-thermal $\mathrm{OH}$ emission is only found within the regions of very dense absorption. The central horse-shoe-shaped cloud has been the most carefully studied. Within the boundaries of the heaviest absorption on the red plates of the Palomar Atlas (the cloud appears quite sharply bounded) are found $\mathrm{OH}$ (Heiles, 1970), formaldehyde (Palmer et al., 1969), and cold neutral hydrogen (Heiles, 1970; Sancisi and Wesselius, 1970; Rohlfs, 1970, private communication). Outside the boundaries of the absorbing cloud, none of these are observed.

The present observations do not allow a decision, whether this is a result of the higher surface density of $\mathrm{OH}$ molecules in these sharply bounded areas of high optical absorption (to which 1 refer to hereafter as 'dark clouds'), or shielding of molecules from UV radiation by heavy dust layers enabling survival for a longer time. We do know that conditions for the formation of low-mass stars are appropriate in these dark clouds. However, the T-Tauri stars are not confined only to the dark clouds. But we cannot decide whether T-Tauri stars can also form in regions of lower absorption, or have been formed originally in dark clouds with most of the dust and gas ending up in stars.

It is interesting to speculate, whether or not a massive dark cloud like the horseshoe-shaped cloud in the Taurus cloud, for which Heiles (1970, private communication) estimates a total mass of about $100 M_{\odot}$, will evolve eventually into a subgroup of an O-star association. If this hypothesis were correct, it should sometime in the future become a strong class $\mathrm{I} \mathrm{OH} / \mathrm{H}_{2} \mathrm{O}$ emission center which some ten thousand years later would turn into a compact $H$ II region such as $G ; 33.9+1.1$ in W 3 .

The evolution of a dark and cool cloud through a T-Tauri star association, class I $\mathrm{OH} / \mathrm{H}_{2} \mathrm{O}$ emission centers and compact $\mathrm{H}$ II regions into an $\mathrm{O}$-star association should be considered as a working hypothesis with many gaps to be filled by future observations. I do not feel that our present observations allow us to conclude, where and why dust and molecules form. The importance, however, of molecular lines as probes of the physical conditions of the interstellar gas and especially of regions of star formation is already clear. I am sure that within the next few years more important information will be obtained that eventually will allow us to solve the problem how stars are formed out of the interstellar matter.

\section{Acknowledgements}

It is my pleasure to thank Lindsey F. Smith of the NASA Goddard Space Flight Center in Greenbelt/Md., USA, for her invaluable help in collecting and sifting the information needed to write this review paper, as well as for many stimulating dis- 
cussions. I further thank G. C. Wynn-Williams, Cambridge, for letting me use his aperture synthesis results prior to publication.

\section{References}

Aikman, G. C.: 1968, Unpublished masters dissertation, University of Toronto.

Blaauw, A.: 1964, Ann. Rev. Astron. Astrophys. 2, 213.

Burke, B. F., Papa, D. C., Papadopoulos, G. D., Schwartz, P. R., Knowles, S. H., Sullivan, W. T., Meeks, M. L., and Moran, J. M.: 1970, Astrophys. J. Letters 160, L63.

Churchwell, E., Felli, M., and Mezger, P. G.: 1969, Astrophys. Letters 4, 33.

Cudaback, D. D. and Heiles, C. E.: 1969, Astrophys. J. Letters 155, L21.

Cudaback, D. D., Read, R. B., and Rougoor, G. W.: 1966, Phys. Rev. Letters 17, 452.

Hayashi, C. and Nakano, T.: 1965, Progr. Theoret. Phys. (Kyoto) 34, 754.

Heiles, C. E.: 1968, Astrophys. J. 151, 919.

Heiles, C. E.: 1969, Astrophys. J. 157, 123.

Heiles, C. E.: 1970, Astrophys. J. 160, 51.

Herbig, G. H.: 1962, Adv. Astron. Astrophys. 1, 47.

Herbig, G. H.: 1964, Trans. IAU XII B, 412.

Herbig, G. H.: 1970, Proc. of the XVIth Liège Symp. 59, 13 (Université de Liège).

Iben, I. and Talbot, R. J.: 1966, Astrophys. J. 144, 968.

Kerr, F. J., Burke, B. F., Reifenstein, E. C. III, Wilson, T. L., and Mezger, P. G.: 1968, Nature $220,1210$.

Litvak, M. M.: 1969, Science.

McCuskey, S. W.: 1938, Astrophys. J. 88, 209.

Mezger, P. G.: 1970a, Proc. of the XVIth Liège Symp. 59, 325 (Université de Liège).

Mezger, P. G.: 1970b, Proc. of the VIth IAU-IUTAM Joint Symposium on Cosmological Gas Dynamics, in press.

Mezger, P. G., Altenhoff, W., Schraml, J., Burke, B. F., Reifenstein, E. C. III, and Wilson, T. L.: 1967, Astrophys. J. Letters 150, L 157.

Mezger, P. G. and Höglund, B.: 1967, Astrophys. J. 147, 490.

Mezger, P. G. and Robinson, B. J.: 1968, Nature 220, 1107.

Mezger, P. G., Wilson, T. L., Gardner, F. F., and Milne, D. K.: 1969, Astron. Astrophys. 4, 96.

Moran, J. M., Burke, B. F., Barrett, A. H., Rogers, A. E. E., Ball, J. A., Carter, J. C., and Cudaback, D. D.: 1968, Astrophys. J. Letters 152, L 97.

Palmer, P., Zuckermann, B., Buhl, D., and Snyder, L. E.: 1969, Astrophys. J. Letters 156, L 47.

Raimond, E. and Eliasson, B.: 1969, Astrophys. J. 155, 817.

Rogers, A. E. E., Moran, J. M., Crowther, P. P., Burke, B. F., Meeks, M. L., Ball, J. A., and Hyde, G. M.: 1966, Phys. Rev. Letters 17, 450.

Ryle, M. and Downes, D.: 1967, Astrophys. J. Letters 148, L 17.

Sancisi, R. and Wesselius, P. R.: 1970, Astron. Astrophys. 7, 341.

Schraml, J. and Mezger, P. G.: 1969, Astrophys. J. 156, 269.

Strand, K. A.: 1958, Astrophys. J. 128, 14.

Turner, B. E.: 1969, Astron. J. 74, 985.

Vandervoort, P. O.: 1964, Astrophys. J. 139, 869.

Webster, W. J. and Altenhoff, W. J.: 1970, Astrophys. Letters 5, 233.

Werner, M. W.: 1970, Astrophys. J, Letters 6, 81.

Williams, I. P. and Cremin, A. W.: 1969, Monthly Notices Roy. Astron. Soc. 144, 359.

\section{DISCUSSION}

Townes: The excitation temperature of $\mathrm{OH}$ may be rather different from the cloud's kinetic temperature. Other evidence gives kinetic temperatures considerably higher than the $5-10^{\circ}$ of $\mathrm{OH}$. Furthermore, there is a collisional mechanism which appears to cool OH below the kinetic temperature in much the same way that $\mathrm{CH}_{2} \mathrm{O}$ is abnormally cooled in the dark clouds.

Sancisi: The anticorrelation of interstellar extinction and $\mathrm{H}$ I emission in the direction of the dense 
Taurus clouds may be explained as due to a local decrease of the spin temperature of the neutral hydrogen connected with the dust. In fact it may indicate that a large amount of cold atomic hydrogen exists in the dust cloud. Molecular hydrogen may also be present but the $21 \mathrm{~cm}$ line results alone are no direct evidence for it, as claimed by Solomon.

A possible association between hydrogen and T-Tauri stars in the area of Taurus was pointed out at the Symposium on 'Pre-Main Sequence Stellar Evolution' held at Liege in 1969. 